\title{
MCSCF-CI Calculations of Radiative Transition Probabilities of PH and PD
}

\author{
J. Senekowitsch, P. Rosmus, and H. J. Werner \\ Fachbereich Chemie der Universität, Niederurseler Hang, D-6000 Frankfurt 50, West Germany
}

M. Larsson

Research Institute of Physics, Frescativägen 24, S-10405 Stockholm, Sweden

Z. Naturforsch. 41 a, 719-723 (1986); received February 21, 1986

Potential energy, dipole moment, and electronic transition moment functions for the $A^{3} \Pi$ and $\mathrm{X}^{3} \Sigma^{-}$states of PH have been calculated from highly correlated electronic wavefunctions. The electric dipole moments in the vibrational ground state of $\mathrm{PH}$ are calculated to be 0.637 Debye $\left(\mathrm{A}^{3} \Pi\right)$ and 0.403 Debye $\left(\mathrm{X}^{3} \Sigma^{-}\right)$. The predicted rates of spontaneous emission between low lying vibrational states of the X state lie in the range of 46 to $109 \mathrm{sec}^{-1}$ (PH) and 12 to $30 \mathrm{sec}^{-1}$ (PD). The calculated radiative lifetime of the $v^{\prime}=0$ level in the $\mathrm{A}^{3} \Pi$ state of $400 \mathrm{~ns}$ is lower by about 10 percent than the most recent experimental value. The classical intersection of the ${ }^{5} \Sigma^{-}$and the $\mathrm{A}^{3} \Pi$ state has been calculated to lie between $v^{\prime}=2$ and 3 with an expected uncertainty of about $500 \mathrm{~cm}^{-1}$, whereas the onset of the rotationally dependent predissociation lies at $v^{\prime}=0, J^{\prime}=11$.

\section{Introduction}

The $\mathrm{A}^{3} \Pi-\mathrm{X}^{3} \Sigma^{-}$electronic transitions in the $\mathrm{PH}$ and $\mathrm{PD}$ radicals have been extensively studied by absorption [1-3] and emission [4] experiments. Due to the predissociation of the A state the pure emission rates, however, are experimentally accessible only for $v^{\prime}=0$. Recently, it was shown that the lifetime in $v^{\prime}=0$ is almost independent of $J^{\prime}$ up to $J^{\prime}=10$, whereas for higher rotational quantum numbers the apparent lifetimes are reduced due to the predissociation of the A state [5]. The average lifetime for $J^{\prime}<10$ was found to be $450 \mathrm{~ns}$, and it is assumed that this corresponds to a pure radiative decay. Previously, two strongly differing lifetimes based on low resolution data have been reported. Since in both experiments the predissociating rotational levels could not be separated, it is to be expected that the observed lifetimes are lower than the pure radiative lifetime. In fact, Sam and Yardley [6] reported, at $6 \AA$ spectral resolution, a lifetime of $355 \mathrm{~ns}$ for the ${ }^{3} \Pi_{1}$ and ${ }^{3} \Pi_{2}$ and $265 \mathrm{~ns}$ for the ${ }^{3} \Pi_{0}$ sublevels. Fink and Welge [7] found an average value of $445 \pm 50 \mathrm{~ns}$ at $10 \AA$ spectral resolution.

In the present work the transition moment function for the $\mathrm{A}^{3} \Pi-\mathrm{X}^{3} \Sigma^{-}$transition has been calculated from highly correlated MCSCF-CI wave-

Reprint requests to Dr. P. Rosmus, Fachbereich Chemie der Universität, Niederurseler Hang, D-6000 Frankfurt 50. functions. After scaling for small remaining errors this function allows evaluating of the radiative transition probabilities of any level in the A state, and it might thus contribute to a detailed understanding of the A state decay mechanism.

Recently, the $v^{\prime \prime}=0$ and 1 levels in the $\mathrm{X}^{3} \Sigma^{-}$state of $\mathrm{PH}$ and PD have been precisely investigated by diode laser [8] and by laser magnetic resonance spectroscopy [9]. To our knowledge, no radiative transition probabilities of the rotation-vibration transitions are so far known from experiments. Also, the dipole moment in the vibrational ground state is experimentally unknown. The MCSCF-CI calculations reported in the present work yield dipole moments with an expected accuracy of about 0.02 Debye and radiative transition probabilities within about 10 percent.

An extended theoretical work on electronically excited states of $\mathrm{PH}$ has been published by Bruna et al. [10]. Those authors also calculated a mean oscillator strength of 0.011 for the $\mathrm{A}^{3} \Pi-\mathrm{X}^{3} \Sigma^{-}$ transition, which corresponds to a lifetime value of $315 \mathrm{~ns}$. This too short value indicates that the correlation contributions to the transition moment have not been fully accounted for (cf. Section III).

Previously, the electronic ground state of $\mathrm{PH}$ has been investigated theoretically by Meyer and Rosmus [11], using PNO-CEPA wavefunctions. The present MCSCF-CI dipole moment function for the

0340-4811 / $86 / 0500-0719 \$ 01.30 / 0$. - Please order a reprint rather than making your own copy. 
$\mathrm{X}$ state is expected to be more accurate and should yield radiative transition probabilities with an accuracy of about 10 percent.

\section{Computational Details and Potential Energy Functions}

The Gaussian-type basis set employed in the calculation consisted of $14 \mathrm{~s}, 11 \mathrm{p}, 4 \mathrm{~d}, 1 \mathrm{f}$ for phosphorus and $8 \mathrm{~s}, 3 \mathrm{p}, 1 \mathrm{~d}$ for hydrogen contracted to $10 \mathrm{~s}, 8 \mathrm{p}$, $4 \mathrm{~d}, 1 \mathrm{f}$ and $6 \mathrm{~s}, 3 \mathrm{p}, 1 \mathrm{~d}$. The phosphorus basis was derived from Veillard's $12 \mathrm{~s}, 10 \mathrm{p}$ set [12] by replacing the 2 most diffuse s-functions by four functions with exponents $0.43,0.21,0.1$ and 0.048 , and the last p-function by two functions with exponents 0.091 and 0.035 . The exponents of the polarization functions were 2.1, 0.7, 0.25 and 0.09 for the d-functions and 0.35 for the f-function. The standard hydrogen $7 \mathrm{~s}$ set [13] was augmented by one function with an exponent of 0.03 . The $\mathrm{p}$ - and d-exponents were 1.2 , $0.3,0.08$, and 0.7 , respectively. Complete active space (CASSCF) [14] and large scale MCSCF [15] calculations were performed in order to select the reference configurations for the final MCSCF-CI [16] calculations. In order to facilitate the calculation of the electronic transition moment, both states were described in a single molecular orbital basis. These orbitals were obtained by minimizing the energy average of the $\mathrm{X}$ and $\mathrm{A}$ states in the MCSCF procedure. With 13 reference configurations for the $\mathrm{X}^{3} \Sigma^{-}$ground state, 23 for the $\mathrm{A}^{3} \Pi$ and 12 for the repulsive ${ }^{5} \Sigma^{-}$state the total number of variational parameters in the internally contracted CI [16] was 33858, 37461, and 20116, respectively. The Schrödinger equation of nuclear motion was solved by the method of Cooley [17].

The present MCSCF-CI wavefunctions are so far the most accurate obtained for $\mathrm{PH}$. The variational energies of the $\mathrm{X}$ and the A state (cf. Table 1) are lower by about 0.03 a.u. than the previous variational energies. In Fig. 1 the potential energy functions for the $\mathrm{X}^{3} \Sigma^{-}, \mathrm{A}^{3} \Pi$ and ${ }^{5} \Sigma^{-}$states are displayed. The spectroscopic constants derived from these potentials are in close agreement with experimental data (cf. Table 1). The equilibrium distances deviate by $0.002 \AA(\mathrm{X})$ and $0.001 \AA(\mathrm{A})$, the harmonic $\omega_{\mathrm{e}}$ constants by $10 \mathrm{~cm}^{-1}$ (X) and $1 \mathrm{~cm}^{-1}$ (A) from the experimental values. Since only very small parts of the potentials can be characterized by the experimental spectroscopic data, the calculated potential energy functions were used in the evaluation of the vibrationally averaged dipole matrix elements. The dissociation energy of the $\mathrm{X}^{3} \Sigma^{-}$state has not yet been accurately determined from spectroscopic data. The onset of predissociation at $J^{\prime}=11$ yields an upper limit of $3.74 \mathrm{eV}$ [5]. This value is about $30 \%$ higher than the theoretical values from the MRD-CI and PNO-CEPA calculations and also higher than the value of $3.17 \mathrm{eV}$ derived from thermochemical cycles [18]. The present MCSCF-CI $D_{\mathrm{e}}$ value of $3.05 \mathrm{eV}$ agrees with the previous theoretical results (cf. Table 1) and is expected to be accurate within $\pm 0.15 \mathrm{eV}$. An even better accuracy has been achieved for the $D_{\mathrm{e}}$ of the A state (cf. Table 1).

Table 1. Calculated spectroscopic data for $\mathrm{PH}$.

\begin{tabular}{|c|c|c|c|c|c|c|c|c|c|c|c|c|}
\hline State & Method & Ref. & $\begin{array}{l}E \\
\text { (a.u.) }\end{array}$ & $\begin{array}{l}R_{\mathrm{e}} \\
(\AA)\end{array}$ & $\begin{array}{l}B_{\mathrm{e}} \\
\left(\mathrm{cm}^{-1}\right)\end{array}$ & $\begin{array}{l}\alpha_{\mathrm{e}} \\
\left(\mathrm{cm}^{-1}\right)\end{array}$ & $\begin{array}{l}\omega_{\mathrm{e}} \\
\left(\mathrm{cm}^{-1}\right)\end{array}$ & $\begin{array}{l}\omega_{\mathrm{e}} x_{\mathrm{e}} \\
\left(\mathrm{cm}^{-1}\right)\end{array}$ & $\begin{array}{l}\mu_{\mathrm{e}} \\
(\mathrm{D})\end{array}$ & $\begin{array}{l}\mu_{\mathrm{e}}^{\prime} \\
(\mathrm{D} / \AA)\end{array}$ & $\begin{array}{l}D_{\mathrm{e}} \\
(\mathrm{eV})\end{array}$ & $\begin{array}{l}T_{\mathrm{e}} \\
\left(\mathrm{cm}^{-1}\right)\end{array}$ \\
\hline \multirow[t]{4}{*}{$X^{3} \Sigma^{-}$} & $\operatorname{MCSCF}(13)-\mathrm{CI}$ & $\begin{array}{l}\text { This } \\
\text { work }\end{array}$ & -341.4260 & 1.423 & 8.524 & 0.253 & 2379 & 42 & 0.431 & -1.30 & 3.05 & \\
\hline & Exp. & {$[4,8,9]$} & & 1.4214 & 8.5327 & 0.2522 & 2366.8 & 45.3 & & & 3.17 & \\
\hline & PNO-CEPA & [11] & -341.4240 & 1.426 & 8.49 & 0.251 & 2366 & 45 & 0.481 & -1.39 & 3.04 & \\
\hline & MRD-CI & {$[10]$} & -341.3984 & 1.4226 & 8.533 & 0.254 & 2380 & 43.9 & & & 3.03 & \\
\hline \multirow[t]{3}{*}{$\mathrm{A}^{3} \Pi$} & $\operatorname{MCSCF}(23)-\mathrm{CI}$ & This & -341.2912 & 1.446 & 8.262 & 0.450 & 2029 & 87 & 0.584 & 1.60 & 0.86 & 29783 \\
\hline & Exp. & {$[4]$} & & $1.4463^{\mathrm{a}}$ & $8.2572^{\mathrm{a}}$ & 0.47 & $2030^{\mathrm{a}}$ & $98.5^{\mathrm{b}}$ & & & $0.89^{c}$ & 29519.6 \\
\hline & MRD-CI & [10] & -341.2565 & 1.4420 & 8.306 & 0.45 & 2071 & 87.8 & & & & 31132.7 \\
\hline
\end{tabular}

a $R_{0}=1.4673 \AA, B_{0}=8.0222 \mathrm{~cm}^{-1}, \Delta G_{1 / 2}=1833 \mathrm{~cm}^{-1}$. - b Estimated from isotope relations.

c Calculated from $D_{\mathrm{e}}\left(\mathrm{X}^{3} \Sigma^{-}\right), T_{\mathrm{e}}$ and $\Delta E\left(\mathrm{P},{ }^{4} \mathrm{~S}-{ }^{2} \mathrm{D}\right)$. 


\section{The $\mathrm{A}^{3} \Pi-\mathrm{X}^{3} \Sigma^{-}$Transition Probabilities}

The calculated MCSCF-CI transition moment function is listed in Table 2 and plotted together with the potential energy functions in Figure 1. The Einstein coefficients of spontaneous emission, radiative lifetimes, and absorption oscillator strengths for the two lowest levels in both electronic states are given in Table 3. The $v^{\prime}=0$ lifetime of $399 \mathrm{~ns}$ is smaller than the experimental value by about $51 \mathrm{~ns}$. As discussed below, this effect is due to the fact that the correlation contributions to the transition moment are very large and probably not fully accounted for.

Very frequently, the Hartree-Fock transition moments of diatomic hydrides are too large, the correlation contributions lie in the range $50-100 \%$. Even if highly correlated electronic wavefunctions are employed, it is possible that this large correlation effect is not fully accounted for. Therefore, in order to check the convergence of the transition moment with respect to the selection of the reference wavefunction, a series of test calculations at $R=2.7$ a.u. has been performed*. The Hartree-Fock calculations yielded total energies of -341.287445 and -341.144274 a.u. for the $\mathrm{X}^{3} \Sigma^{-}$and the $\mathrm{A}^{3} \Pi$ state, respectively, and a transition moment of 0.588 a.u. In all further calculations the $1 \mathrm{~s}, 2 \mathrm{~s}$ and $2 p$ shells of the phosphorus atom were treated as core and taken from the previous Hartree-Fock calculation. In order to select the reference configurations for the MCSCF-CI procedure, state averaged complete active space (CASSCF) calculations with different active orbital sets have been performed. This yielded 13 configurations for the $\mathrm{X}^{3} \Sigma^{-}$state and 23 configurations for the A ${ }^{3} \Pi$ state in the orbital space $4 \sigma, 5 \sigma, 6 \sigma, 2 \pi, 3 \pi$, and $1 \delta$. The increased number of configurations for the $\mathrm{A}^{3} \Pi$ state is demanded by the averaging procedure. In particular, the reference wavefunction for the A state was made invariant with respect to rotations between the $4 \sigma$ and $5 \sigma$ orbitals in order to achieve a balanced description of the strongly interacting states with the dominant configurations $4 \sigma^{2} 5 \sigma$ and $4 \sigma 5 \sigma^{2}$. Further investigations with CASSCF wavefunctions also showed considerable amounts of $\delta$-type orbital contribution. Although the $\mathrm{CI}$ energy was nearly unaffected by additional reference configurations with these orbitals, they considerably changed the transition moment*. For that reason $\delta$-type orbitals have been included into the final reference set used in the present calculations. This leads to MCSCF energy values of -341.324883 and -341.178804 a.u. for the $\mathrm{X}^{3} \Sigma^{-}$ and $\mathrm{A}^{3} \Pi$ states, respectively, and a transition moment of 0.308 a.u. The MCSCF-CI procedure yielded - 341.426902 a.u., -341.291054 a.u., and 0.234 a.u., respectively. Calculations with even larger configuration sets did not result in considerable changes. Nevertheless the calculated lifetime of $399 \mathrm{~ns}$ is too short compared to the most recent experimental value. Thus we conclude that the remaining error in the transition moment of about $5-6 \%$ is due to missing parts of the large correlation contribution of more than 0.35 a.u. relative to the Hartree-Fock transition moment. Similar errors have been found for other diatomic hydrides like $\mathrm{OH}, \mathrm{HCl}^{+}$[19] and $\mathrm{SH}$ [20]. If the electronic transition moment function is shifted by only 0.014 a.u. to lower values, a lifetime of $450 \mathrm{~ns}$ in $v^{\prime} \times 0$ is obtained. Thus, we recommend a linear shift of the function in Table 2 for the evaluation of

Table 2. Dipole and transition moment functions for $\mathrm{PH}$ (all values in a.u.).

\begin{tabular}{lrrr}
\hline Distance & $\left\langle\mathrm{A}^{3} \Pi\left|\frac{x+i y}{\sqrt{2}}\right| \mathrm{X}^{3} \Sigma^{-}\right\rangle$ & $\mathrm{X}^{3} \Sigma^{-}$ & $\mathrm{A}^{3} \Pi$ \\
\hline 2.0 & 0.3249 & 0.3181 & 0.0244 \\
2.4 & 0.2774 & 0.2402 & 0.1229 \\
2.6 & 0.2490 & 0.1929 & 0.1854 \\
2.7 & 0.2338 & 0.1666 & 0.2182 \\
3.0 & 0.1852 & 0.0828 & 0.3192 \\
3.3 & 0.1342 & -0.0029 & 0.3831 \\
3.6 & 0.0841 & -0.0835 & 0.3612 \\
4.0 & 0.0269 & -0.1580 & 0.2220 \\
4.5 & -0.0185 & -0.1835 & 0.0509 \\
\hline
\end{tabular}

Table 2 a. Transition moment in atomic units at $R=2.7$ a.u.

\begin{tabular}{ll}
\hline Method & Transition moment \\
\hline Hartree-Fock & $0.588^{\mathrm{a}}$ \\
CASSCF & $0.409^{\mathrm{b}, \mathrm{d}}$ \\
CASSCF & $0.245^{\mathrm{c}, \mathrm{d}}$ \\
MCSCF $(13 / 23)$ & $0.255^{\mathrm{c}, \mathrm{d}}$ \\
MCSCF $(13 / 23)-C I$ & $0.234^{-C}$ \\
\hline
\end{tabular}

a States optimized separately.

b Active orbitals $4 \sigma 5 \sigma 6 \sigma 2 \pi 3 \pi$.

c Active orbitals $4 \sigma 5 \sigma 6 \sigma 2 \pi 3 \pi 1 \delta$.

d Single set of orthogonal molecular orbitals optimized by minimizing the energy average of both states.

* cf. Table 2 a. 
Table 3. Einstein coefficients of spontaneous emission, radiative lifetimes and absorption oscillator strengths for the $\mathrm{A}^{3} \Pi-\mathrm{X}^{3} \Sigma^{-}$transition in PH and PD.

\begin{tabular}{llllllll}
\hline Species & $v^{\prime}$ & $\mathrm{A}_{0}^{v^{\prime}}\left(\mathrm{s}^{-1}\right)$ & $\mathrm{A}_{1}^{v^{\prime}}\left(\mathrm{s}^{-1}\right)$ & $\mathrm{A}_{2}^{v^{\prime}}\left(\mathrm{s}^{-1}\right)$ & $\tau(\mathrm{ns})$ & $f_{v^{\prime} 0}$ & $f_{v^{\prime} 1}$ \\
\hline $\mathrm{PH}$ & 0 & $2.49 \cdot 10^{7}$ & $1.35 \cdot 10^{5}$ & $7.97 \cdot 10^{2}$ & 399 & $8.6 \cdot 10^{-3}$ & $5.5 \cdot 10^{-5}$ \\
& 1 & $3.16 \cdot 10^{5}$ & $1.74 \cdot 10^{7}$ & $4.98 \cdot 10^{4}$ & 473 & $9.7 \cdot 10^{-4}$ & $6.2 \cdot 10^{-3}$ \\
$\mathrm{PD}$ & 0 & $2.52 \cdot 10^{7}$ & $3.24 \cdot 10^{5}$ & $1.53 \cdot 10^{3}$ & 390 & $8.7 \cdot 10^{-3}$ & $1.3 \cdot 10^{-4}$ \\
& 1 & $3.08 \cdot 10^{5}$ & $1.87 \cdot 10^{7}$ & $8.77 \cdot 10^{4}$ & 439 & $9.7 \cdot 10^{-4}$ & $6.6 \cdot 10^{-3}$ \\
\hline
\end{tabular}

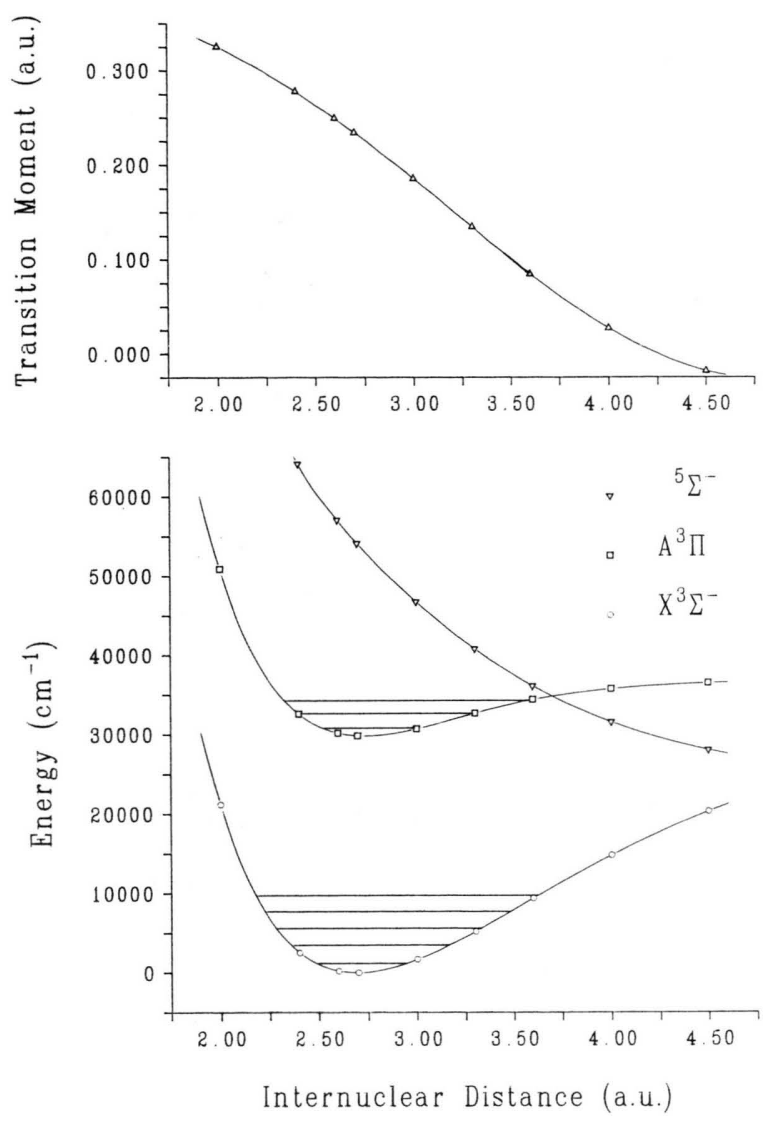

Fig. 1. MCSCF-CI potential energy and electronic transition moment functions for the $\mathrm{X}^{3} \Sigma^{-}, \mathrm{A}^{3} \Pi$ and ${ }^{5} \Sigma^{-}$states of $\mathrm{PH}$.

A- $\mathrm{X}$ radiative transition probabilities in possible future applications.

The radiative lifetimes in the $\mathrm{A}$ state increase with increasing vibrational quantum numbers, but since the nonradiative decay is expected to be much faster, only values for $v^{\prime}=0$ and 1 are listed in Table 3. The deuterium substitution lowers the lifetime in $v^{\prime}=0$ by about 2 percent, in $v^{\prime}=1$ by about 7 percent.

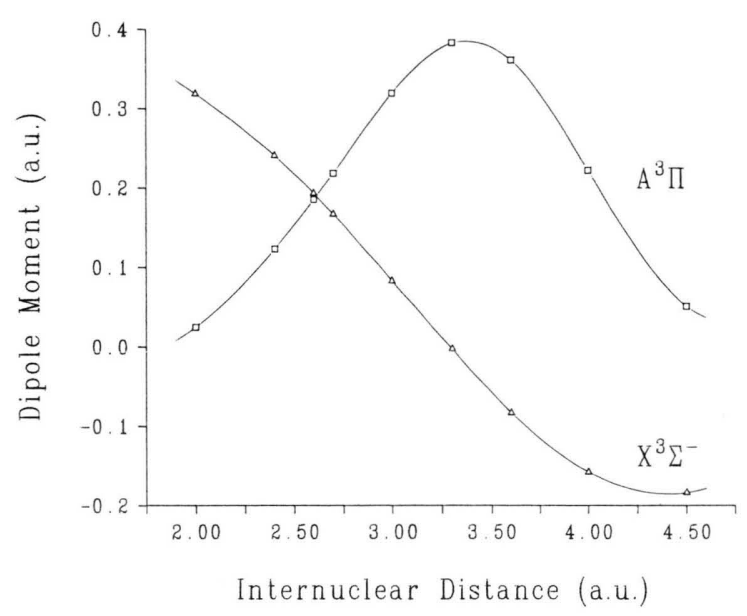

Fig. 2. MCSCF-CI electric dipole moment functions of the $\mathrm{X}^{3} \Sigma^{-}$and $\mathrm{A}^{3} \Pi$ states of $\mathrm{PH}$.

The predissociation of the A state is due to the intersection with the ${ }^{5} \Sigma^{-}$state correlating with the lowest dissociation asymptote $\mathrm{P}\left({ }^{4} \mathrm{~S}\right)+\mathrm{H}\left({ }^{2} \mathrm{~S}\right)$. The intersection is calculated at $R=3.6$ a.u., i.e. between the outer classical turning points of $v^{\prime}=2$ and 3 . We expect a relative error in our calculated total energies of about $500 \mathrm{~cm}^{-1}$. In order to describe the dissociative process quantitatively the $R$ dependence of the ${ }^{5} \Sigma^{-}-{ }^{3} \Pi$ coupling and the possible tunneling from the low lying levels of the A state would have to be calculated. In this connection it would also be of interest to measure the differences in the lifetimes of $\mathrm{PH}$ versus PD.

\section{The Dipole Moments and Radiative Transition Probabilities in the $\mathrm{X}^{3} \Sigma^{-}$State}

The MCSCF-CI dipole moments calculated as expectation values for the $\mathrm{X}$ and $\mathrm{A}$ states are listed in Table 2 and plotted in Figure 2. The $\mu_{\mathrm{e}}$ value of 0.431 Debye is lower by 0.05 Debye than the PNOCEPA value (cf. Table 1). Similar deviations 
Table 4. Vibrational electric dipole matrix elements (Debye) and rates of spontaneous emission $\left(\mathrm{sec}^{-1}\right)$.

\begin{tabular}{lllllllr}
\hline Species & State & $v$ & $R_{v}^{v}$ & \multicolumn{1}{c}{$R_{v}^{v+1}$} & \multicolumn{1}{c}{$R_{v}^{v+2}$} & $A_{v}^{v+1}$ & \multicolumn{1}{c}{$A_{v}^{v+2}$} \\
\hline PH & $\mathrm{X}^{3} \Sigma^{-}$ & 0 & 0.403 & -0.111 & 0.006 & 45.8 & 0.9 \\
& & 1 & 0.355 & -0.160 & 0.009 & 81.2 & 2.0 \\
& & 2 & 0.312 & -0.199 & 0.016 & 109.8 & 5.4 \\
& $\mathrm{~A}^{3} \Pi$ & 0 & 0.638 & 0.143 & -0.034 & 40.8 & 14.6 \\
$\mathrm{PD}$ & & 1 & 0.714 & 0.169 & -0.080 & 36.8 & 47.5 \\
& $\mathrm{X}^{3} \Sigma^{-}$ & 0 & 0.410 & -0.094 & 0.004 & 12.7 & 0.2 \\
& & 1 & 0.373 & -0.135 & 0.006 & 23.0 & 0.3 \\
& & 2 & 0.344 & -0.168 & 0.009 & 31.4 & 0.7 \\
& $\mathrm{~A}^{3} \Pi$ & 0 & 0.626 & 0.124 & -0.021 & 12.7 & 2.5 \\
& & 1 & 0.687 & 0.163 & -0.047 & 16.8 & 9.3 \\
\hline
\end{tabular}

between both theoretical approaches have been found for $\mathrm{SH}$ [20], and also between the experimental and PNO-CEPA values for $\mathrm{HCl}$. From this systematic behavior it can be concluded that the previous CEPA results yielded somewhat too large dipole moments and we expect that the present MCSCF-CI values are accurate within about 0.02 Debye. In $v^{\prime \prime}=0$ of $\mathrm{PH}$ the electric dipole moment amounts to 0.403 Debye, for $\mathrm{PD}$ to 0.409 Debye. This small increase is due to the decrease of the dipole moment function close to the equilibrium distance. For the $\mathrm{A}$ state the first

[1] R. W. B. Pearse, Proc. Roy. Soc. London A 129, 328 (1930).

[2] M. Ishaque and R. W. B. Pearse, Proc. Roy. Soc. London A 173, 265 (1939).

[3] F. Legay, Can. J. Phys. 38, 797 (1960).

[4] J. Rostas, D. Cossart, and J. R. Bastien, Can. J. Phys. 52, 1274 (1974).

[5] O. Gustafsson, G. Kindvall, M. Larsson, J. Senekowitsch, and P. Sigray, Mol. Phys. 56, 1369 (1985).

[6] C. L. Sam and J. T. Yardley, J. Chem. Phys. 69, 4621 (1978).

[7] E. Fink and K. H. Welge, Z. Naturforsch. 19a, 1193 (1964).

[8] J. R. Anaconda, P. B. Davies, and P. A. Hamilton, Chem. Phys. Lett. 104, 269 (1984).

[9] N. Ohashi, K. Kawaguchi, and E. Hirota, J. Molec. Spectr. 103, 337 (1984).

[10] P. J. Bruna, G. Hirsch, S. D. Peyerimhoff, and R. J. Buenker, Mol. Phys. 42, 875 (1981).

[11] W. Meyer and P. Rosmus, J. Chem. Phys. 63, 2356 (1975). derivative of $\mu\left(R_{\mathrm{e}}\right)$ is positive (cf. Figure 2), and the $\mu_{0}$ value is smaller for PD than for $\mathrm{PH}$ (cf. Table 4). The $\mu_{0}$ in the A state of $\mathrm{PH}$ has been calculated to be 0.638 Debye. In the $X$ state the diagonal dipole matrix elements decrease with increasing vibrational quantum numbers, in the $\mathrm{A}$ state they increase. The rather linear shape of the $\mathrm{X}$ state function leads to large absorption intensities and emission rates for the fundamental sequence and small intensities for the overtones. Since the A state function has a maximum close to the equilibrium distance, the overtones within the A state are stronger than in the ground state (cf. Table 4).

The $\mathrm{X}$ state radiative transition probabilities are of the same magnitude as in $\mathrm{HCl}$ [19], and there should be a good chance to observe infrared emission in PH and PD. Such experiments would help to characterize higher vibrational states of $\mathrm{PH}$, which have not yet been investigated experimentally.

\section{Acknowledgements}

This work was supported by the Deutsche Forschungsgemeinschaft and Fonds der Chemischen Industrie.

[12] A. Veillard, Theo. Chim. Acta 12, 405 (1968).

[13] S. Huzinaga, Technical Report: Approximate Atomic Functions, Division of Theoretical Chemistry, University of Alberta.

[14] H.-J. Werner and P. J. Knowles, J. Chem. Phys. 82, 5053 (1985); P. J. Knowles and H.-J. Werner, Chem. Phys. Lett. 115, 259 (1985).

[15] H.-J. Werner and W. Meyer, J. Chem. Phys. 73, 2342 (1980); 74, 5794 (1981).

[16] H.-J. Werner and E. A. Reinsch, J. Chem. Phys. 76, 3144 (1982).

[17] J. W. Cooley, Math. Comp. 15, 363 (1961).

[18] G. Di Stefano, M. Lenzi, A. Margani, A. Mele, and C. N. Xuan, J. Photochem. 7, 335 (1977).

[19] H.-J. Werner, P. Rosmus, W. Schätzel, and W. Meyer, J. Chem. Phys. 80, 831 (1984).

[20] J. Senekowitsch, H.-J. Werner, P. Rosmus, E. A. Reinsch, and S. V. O'Neil, J. Chem. Phys. 83, 4661 (1985). 